17

\title{
Спектрально улучшенная квантовая память на контролируемой частотной гребенке
}

\author{
(ㄱ Н.С. Перминов ${ }^{1,2}$, Д.Ю. Таранкова ${ }^{3}$, С.А. Моисеев ${ }^{1,2, \text { ॠ }}$ \\ ${ }^{1}$ Казанский квантовый центр, Казанский национальный исследовательский технический университет \\ им. А.Н. Туполева-КАИ, \\ 420111 Казань, Россия \\ ${ }^{2}$ Казанский физико-технический институт им. Е.К. Завойского, \\ 420029 Казань, Россия \\ ${ }^{3}$ Кафедра радиоэлектроники и информационно-измерительной техники, \\ Казанский национальный исследовательский технический университет им. А.Н. Туполева - КАИ, \\ 420111 Казань, Россия \\ ฯ e-mail: s.a.moiseev@kazanqc.org \\ Поступила в редакцию 11.07.2018 г. \\ В окончательной редакции 12.04.2019 г. \\ Принята к публикации 16.04.2019 г.
}

\begin{abstract}
Предложена схема блока квантовой памяти, состоящего из трех кольцевых микрорезонаторов, обладающих разными частотами и взаимодействующих последовательно друг с другом и с общим волноводом. Найдены параметры микрорезонаторов, показывающие возможность высокоэффективного сохранения световых полей на данном блоке памяти при оптимальном выборе параметров гребенки частот микрорезонатров и найдена процедура оптимальной склейки нескольких блоков памяти, которая позволяет увеличивать ширину рабочей полосы квантовой памяти при сохранении высокой эффективности.
\end{abstract}

Ключевые слова: квантовая информатика, квантовая память, контролируемая частотная гребенка, кольцевые резонаторы, оптимизация спектра.

DOI: $10.21883 /$ OS.2019.08.48048.202-18

\section{Введение}

Динамика многочастичных систем, обладающих большим количеством управляемых параметров [1,2], дает широкие возможности для конструирования композитных резонансных схем с наперед заданными спектральными свойствами. Значительные успехи на этом пути достигнуты при управлении световыми полями в системе взаимосвязанных высокодобротных резонаторов, позволяющих создавать световые поля с заданным пространственным распределением, получивших название „фотонных молекул“ [3]. Такого типа многорезонаторные (MP) системы успешно разрабатываются в качестве управляемых линий задержки [4-6], интегрированных в оптоволоконные линии.

Появление высокодобротных резонаторов [7] и их интеграция в МР структуры [8] делает их интересными для использования в схемах оптической и микроволновой квантовой памяти (КП) [9-11], в которых резонаторы могут иметь разные частоты. Такие схемы демонстрируют возможность значительного увеличения рабочего спектрального диапазона КП, а высокая добротность резонаторов позволяет значительно усиливать постоянные связи как между соседними резонаторами, так и с находящимися в резонаторах атомными системами. Последнее свойства делают данного типа КП перспективной для использования в схемах универсального квантового компьютера [12-15].
В настоящей работе разработана новая схема КП, состоящая из последовательно соединенных компактных блоков КП, каждый из которых содержит несколько связанных друг с другом резонаторов. Найдены оптимальные параметры резонаторов в блоке, их связь с другом, а также разработана процедура интегрирования блоков в общую волноводную схему КП, при которой возможно достижение высокой квантовой эффективности сохранения световых полей в спектральном диапазоне, на порядки превосходящем спектральную ширину отдельных резонаторов и каждого блока памяти. Обсуждена возможность предлагаемой схемы КП для ее непосредственного использования при комнатной температуре.

\section{Физическая модель}

Исходная идея рассматриваемой схемы опирается на подход к КП, основанный на технике фотонного эха [1618] в варианте, использующем резонансные атомы с периодической спектральной структурой неоднородного уширения линии поглощения, который известен как AFC-протокол [19], а также на реализации данного подхода в оптимальном резонаторе [20,21]. В отличие от [19] в изучаемых нами МР схемах вместо атомной системы используется система из микрорезонаторов, связанных с общим волноводом $[9,10]$ или волноводным 


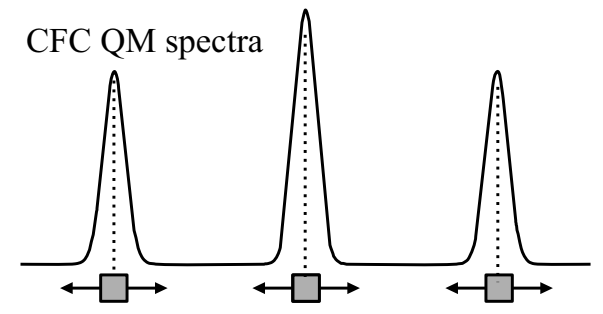

- controllable couplings and frequencies

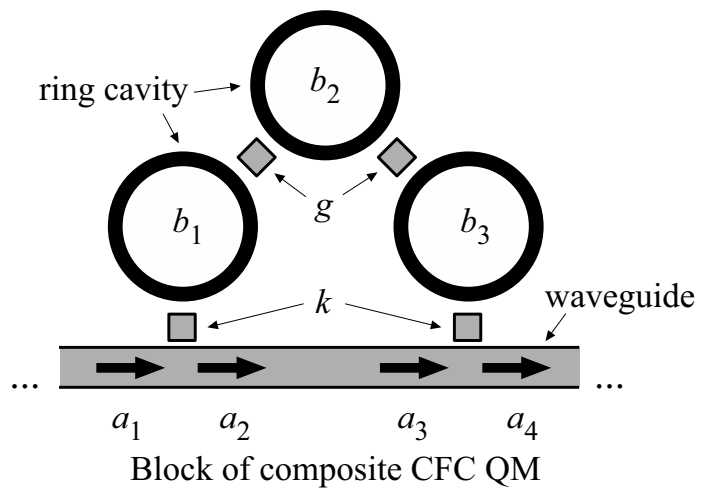

Pис. 1. Принципиальная схема блока композитной КП на контролируемой частотной гребенке из кольцевых микрорезонаторов (controllable frequency comb quantum memory CFC QM).

резонатором [11], что открывает возможность создания широкополосного высокоэффективного интерфейса на конечном числе резонаторов.

Каждый блок рассматриваемой схемы КП на контролируемой частотной гребенке (controllable frequency comb - CFC), изображенный на рис. 1, состоит из трех последовательно соединенных кольцевых микрорезонаторов, обладающих разными частотами и взаимодействующих с общим волноводом через первый и третий микрорезонаторы. Внутри блока геометрическое расположение микрорезонаторов вдоль волновода и друг относительно друга выбрано так, что при прохождении света от первого резонатора к третьему через волновод или через второй микрорезонатор оптический путь кратен целому числу полуволн и не возникает дополнительного фазового набега. Также предполагаем, что необратимые потери в резонаторах пренебрежимо малы на рассматриваемых временах и соблюдается симметрия распределения спектроскопических параметров (частот, констант связи микрорезонаторов друг с другом и волокном) относительно второго микрорезонатора внутри каждого блока. Последнее условие является естественным требованием для универсальных блоков КП, из которых можно было бы составить общую КП с произвольной рабочей спектральной шириной. Мы изучаем возможности спектрального улучшения рассматриваемых блоков КП за счет оптимизации их спектроскопических параметров и постоянной связи, а также рассматриваем процедуру оптимальной склейки блоков памяти, которая позволяет увеличивать ширину рабочей полосы КП при сохранении общей высокой квантовой эффективности.

Используя известный формализм квантовой оптики [22] для описания взаимодействия однофотонных световых полей в волноводе и резонаторах [9,20], получим следующие уравнения для входных $a_{1}(t), a_{3}(t)$ и выходных $a_{2}(t), a_{4}(t)$ световых мод в волноводе (рис. 1) и мод кольцевых микрорезонаторов $b_{1}(t), b_{2}(t), b_{3}(t)$ :

$$
\begin{gathered}
{\left[\partial_{t}+i \Delta_{1}+k_{1} / 2+\gamma_{1} / 2\right] b_{1}(t)+i g_{1} b_{2}(t)} \\
=\sqrt{k_{1}} a_{1}(t)+\sqrt{\gamma_{1}} F_{1}(t), \\
{\left[\partial_{t}+i \Delta_{2}+\gamma_{2} / 2\right] b_{2}(t)+i g_{1}^{*} b_{1}(t)+i g_{2}^{*} b_{3}(t)=\sqrt{\gamma_{2}} F_{1}(t),} \\
{\left[\partial_{t}+i \Delta_{3}+k_{2} / 2+\gamma_{3} / 2\right] b_{3}(t)+i g_{2} b_{2}(t)} \\
=\sqrt{k_{2}} a_{3}(t)+\sqrt{\gamma_{3}} F_{1}(t), \\
a_{1}(t)-a_{2}(t)=\sqrt{k_{1}} b_{1}(t), \\
a_{3}(t)-a_{4}(t)=\sqrt{k_{2}} b_{3}(t), \\
a_{3}(t)=a_{2}(t),
\end{gathered}
$$

где $\left\{\Delta_{1}=-\Delta, \Delta_{2}=0, \Delta_{3}=\Delta\right\}-$ отстройки микрорезонаторов от центральной частоты $v_{0},\left\{g_{1}=g\right.$, $\left.g_{2}=g\right\}$ - константы связи между микрорезонаторами, $\left\{k_{1}=k, k_{2}=k\right\}$ - константы связи между микрорезонаторами и волноводом, $\left\{\gamma_{1}, \gamma_{2}, \gamma_{3}\right\}$ - декременты затухания поля в резонаторах, $\sqrt{\gamma_{n}} F_{n}(t)$ - дельтакоррелированные силы Ланжевена [3,22,23]:

$$
\left(\left\langle F_{n}^{\dagger}(t)\right\rangle=\left\langle F_{n}(t)\right\rangle=0, \quad\left\langle F_{n}(t) F_{n}^{\dagger}\left(t^{\prime}\right)\right\rangle=\delta\left(t-t^{\prime}\right)\right),
$$

вкладом которых в дальнейшем пренебрегаем ввиду малой константы взаимодействия оптического кванта с фононами внутри микрорезонаторов [3,23], т.е. $\gamma_{n} t_{\text {storage }} \sim \gamma_{n} / \Delta \ll 1$ влечет уменьшение вклада $\sqrt{\gamma_{n}} F_{n}(t)$ в квантовую динамику на рассматриваемых временах $t_{\text {storage }} \sim 1 / \Delta[23]$. Для частного случая, когда потери $\gamma_{n}$ обусловлены отрывом циркулирующего поля от резонаторов и могут быть представлены распадом поля во внешний вакуум [3], силы Ланжевена не вносят вклад в эффективность. Для исследуемой системы начальное и конечное квантовые состояния описываются как

$$
\left|\Psi_{\text {in,out }}\right\rangle=\int d t e^{-i v_{0} t} a_{1,4}^{\dagger}(t)|0\rangle
$$

а ненулевые коммутационные соотношения для операторов $\left\{a_{n}(t), b_{n}(t)\right\}$ имеют вид

$$
\left[a_{n}(t), a_{n}^{\dagger}\left(t^{\prime}\right)\right]=\delta\left(t-t^{\prime}\right), \quad\left[b_{n}(t), b_{n}^{\dagger}(t)\right]=1 .
$$

Используя фурье-преобразование, из системы уравнений (1) находим выходное поле $a_{4}(t)$ через передаточную функцию (ПФ) $S(v)$, определяемую через 
соотношение для операторов $\tilde{a}_{4}(v)=S(v) \tilde{a}_{1}(v)$ :

$$
\begin{gathered}
S(v)=\exp \{i v T(v)\}, \\
T(v)=\operatorname{Arctan}\left(4 k v /\left[8 g^{2}+k^{2}+4-4 v^{2}\right]\right) / v,
\end{gathered}
$$

где

$$
a_{1,4}(t)=(2 \pi)^{-1 / 2} \int d v e^{-i v t} \tilde{a}_{1,4}(v),
$$

$T(v)$ - функция времени задержки (длительности памяти) от частоты $v$, а также без ограничения общности рассуждений мы положили $\Delta=1$ (здесь и далее все величины даны в единицах $\Delta$ ). В нашей ситуации для однофотонного уровня передаточная функция уже не является оператором, но определяет все характеристики системы (эффективность и фиделити) и далее будем оптимизировать $S(v)$.

\section{Оптимизация блока КП}

Как видно из (2), спектральная эффективность $\eta(v)=|S(v)|^{2}$ исследуемой модельной КП в любой полосе частот $v$ равна 1 и основные физические характеристики памяти содержатся в спектральной функции задержки $T(v)$, свойства которой однозначно определяют точность восстановления входного сигнала произвольной формы. При этом следует помнить, что оптимизация точности восстановления для данного конкретного входного сигнала $a_{1}(t)$ и оптимизация функции $T(v)$ в широкой полосе частот (для входного сигнала $a_{1}(t)$ произвольной формы) являются разными задачами. Идеальная бесконечно широкая по спектру КП характеризуется условием $T_{\mathrm{rel}}(v)=T(v) / T(0)=1$ для любой частоты $v$, которое не может быть достигнуто ввиду конечности числа поглотителей. Для системы с финитным числом поглотителей последнее условие нужно заменить приближенным равенством $T(v) \approx T(0)$, которое эквивалентно оптимизации величины $|T(v)-T(0)|^{2}$ в широкой полосе частот [14]. Физически это означает, что функция $T(v)$ имеет плато в спектре (область малых изменений функции) в некоторой окрестности $v=0$, которое за счет оптимизации можно сделать более плоским и широким (увеличить соответственно эффективную полосу памяти).

Согласно [14], для КП с $Q$ свободными параметрами и $N_{0}$ резонаторами, касающимися волновода, для оптимизации (минимизации) выбирается целевая функция $H=\sum_{m=-N_{0} / 2}^{N_{0} / 2}\left|T\left(v=2 m / N_{0}\right)\right|^{2}$, отвечающая оптимизации в полосе шириной порядка $N_{0} \Delta(\Delta=1)$. Случай наличия двух свободных параметров $k, g$ далее мы будем называть полной оптимизацией, а случай наличия только одного свободного параметра $k-$ частичной оптимизацией $(g=0$ и в каждом блоке вспомогательный резонатор, не взаимодействующий напрямую с волноводом, фактически отсутствует в системе).

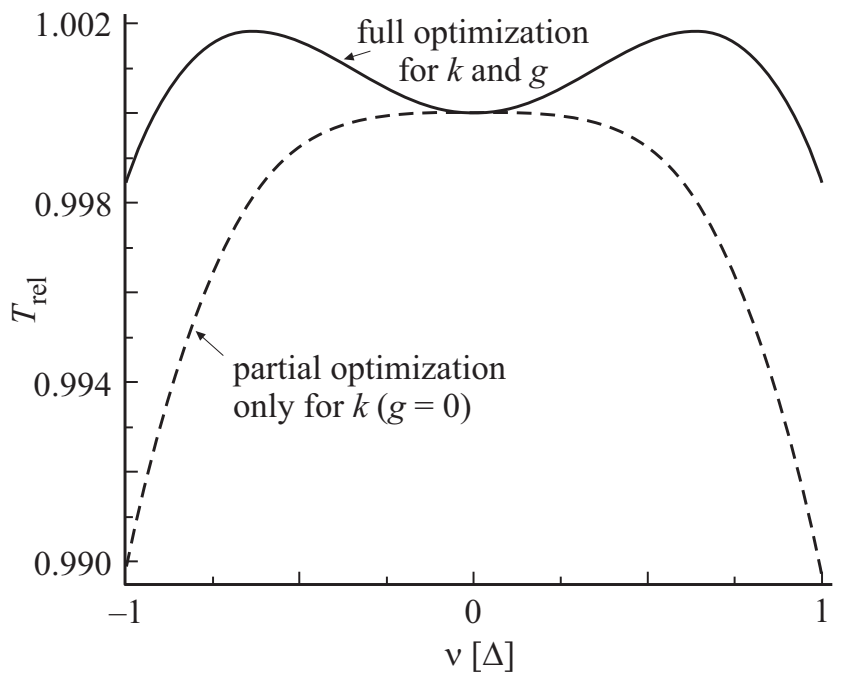

Рис. 2. Нормированное время задержки $T_{\text {rel }}$ в зависимости от частоты $v$ для оптимизированного блока КП с выключенной $\{g=0, k=3.17\}$ (частичная оптимизация - штриховая линия) и включенной связью $\{g=0.29, k=3.47\}$ (полная оптимизация - сплошная линия). Разброс времени задержки для сплошной кривой 0.004 против 0.006 для штриховой кривой, соответствующей только частичной оптимизации.

Результаты оптимизации одного блока КП изображены на рис. 2, где показано нормированное время задержки $T_{\text {rel }}$ в зависимости от частоты $v$ для частично оптимизированного блока КП с выключенной связью $\{g=0, k=3.17\}$ и полностью оптимизированного блока с включенной связью $\{g=0.29, k=3.47\}$. Разброс времени задержки внутри частотной полосы $[-\Delta ; \Delta]$ для полной оптимизации составляет 0.004 против 0.006 для частичной оптимизации. Физически это означает, что подключение дополнительного микрорезонатора к блоку многорезонаторной КП [9] из двух микрорезонаторов позволяет в 1.5 раза уменьшить спектральные ошибки в рабочей полосе частот или использовать в 1.5 раза более широкую полосу частот при сохранении прежней спектральной точности.

\section{Процедура склейки блоков}

Для существенного увеличения спектральной ширины полосы КП мы предлагаем расположить вдоль волноводной линии несколько универсальных блоков КП, каждый из которых будет покрывать свою полосу частот, а соседние полосы будут перекрываться друг с другом оптимальным образом. Таким образом, ширина рабочей полосы КП может быть увеличена на несколько порядков. Мы продемонстрируем процедуру оптимальной спектральной склейки двух полностью оптимизированных блоков КП (всего 6 резонаторов, 4 из них соединены с волноводом), частотные центры которых приходятся на частоты $-d$ и $d$, и сравним 


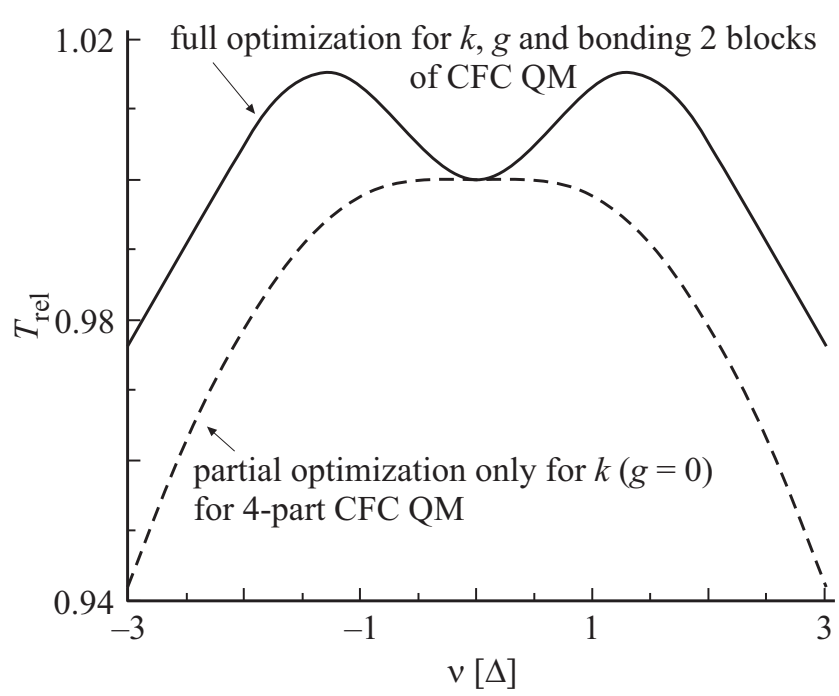

Рис. 3. Нормированное время задержки $T_{\text {rel }}$ в зависимости от частоты $v$ после выполнения процедуры оптимальной склейки двух блоков КП с выключенной связью $\{g=0, k=4.26\}$, задействованы только четыре резонатора (частичная оптимизация - штриховая линия), и включенной связью $\{d=2.18\}$ (полная оптимизация - сплошная линия). Разброс времени задержки для штриховой кривой 0.06 , а для сплошной кривой 0.04 , что отвечает лучшему спектральному качеству КП.

полученную схему с ранее предложенной для случая 4 резонаторов, соединенных с волокном, частоты которых отстроены эквидистантно, а константа связи каждого резонатора с волноводом равна $k$ [9]. Максимальное отклонение при склейке блоков возникает в точке спектра посередине между центрами частот каждого из блоков, поэтому выбор оптимального расстояния $d$ для склейки двух уже полностью оптимизированных блоков КП осуществлялся исходя из простого правила $T(v=-d)=T(v=0)=T(v=d)$, которое надежно гарантирует однородность всей конечной области. Выбор оптимального параметра $k$ для случая эквидистантно расположенных по спектру резонаторов осуществлялся на основе оптимизации величины $|T(v)-T(0)|^{2}$ согласно [14], что сводится к решению уравнения $\left.\partial_{v}^{2} T(v)\right|_{\nu=0}=0$ относительно $k$.

В результате мы получили $d=2.18$ для склейки оптимальных блоков КП и $k=4.26$ для случая четырех резонаторов. Как видно из рис. 3, разброс времени задержки внутри частотной полосы $[-3 \Delta ; 3 \Delta]$ для случая четырех резонаторов составляет 0.06 , а для двух склеенных универсальных блоков КП эта величина равна 0.04, что соответствует лучшему спектральному качеству композитной КП. Аналогичная процедура склейки блоков может быть выполнена и для большего числа блоков при помощи методов оптимизации, предложенных в [14], что позволяет дополнительно улучшить качество и величину ширины рабочей полосы частот конечной композитной КП.

\section{Заключение}

Предложенная схема композитной КП позволяет с высокой точностью оптимизировать рабочий спектр широкополосной квантовой памяти на контролируемой частотной гребенке из спектральных линий кольцевых микрорезонаторов, взаимодействующих друг с другом и с общим волноводом. Удобство контроля характеристик предложенной многорезонаторной схемы КП обусловлено тем, что она состоит из предварительно оптимизированных по спектральным характеристикам блоков, каждый из которых может быть дополнительно подключен/отключен независимо от остальных. При этом процедура склейки блоков позволяет на несколько порядков увеличить ширину рабочей полосы конечной композитной КП при сохранении высокой эффективности.

Изученная схема КП может быть реализована как в оптическом, так и в микроволновом диапазоне на основе существующих технологий $[6,7,10]$ и представляет большой интерес для создания практически значимого многокубитового квантового интерфейса и КП для универсального квантового компьютера. Примечательным свойством такой КП оптического диапазона является возможность ее непосредственного использования при комнатной температуре в силу большой энергии оптического кванта. При этом важным обстоятельством является разработка способа динамического управления временем сохранения светового поля. Это возможно за счет отключения резонаторов от волновода на заданное время, однако изучение этого вопроса требует дополнительных исследований.

\section{Финансирование работы}

Работа частично поддержана грантом Российского научного фонда № 14-12-01333-П (основная идея и анализ результатов - С.А.М., Н.С.П.), а также частично поддержана в рамках бюджетной темы Лаборатории квантовой оптики и информатики Казанского физикотехнического института им. Е.К. Завойского - обособленного структурного подразделения ФИЦ КазНЦ РАН грант № 0217-2018-0005 (численное моделирование Н.С.П., Т.Д.Ю.).

\section{Конфликт интересов}

Авторы заявляют, что у них нет конфликта интересов.

\section{Список литературы}

[1] Hartmann M.J., Brandao F.G.S.L., Plenio M.B. // Las. Photonics Rev. 2008. N 2. P. 527.

[2] Roy D., Wilson C.M., Firstenberg O. // Rev. Mod. Phys. 2017. V. 89. N 2. P. 021001.

[3] Li Y., Abolmaali F., Allen K.W., Limberopoulos N.I., Urbas A., Rakovich Yu., Maslov A.V., Astratov V.N. // Las. Photonics Rev. 2017. V. 11. N 2. P. 1600278. 
[4] Heebner J.E., Boyd R.W. // J. Mod. Opt. 2002. V. 49. N 14-15. P. 2629.

[5] Xia F., Sekaric L., Vlasov Y. // Nat. Photon. 2007. V. 1. N 1. P. 65.

[6] Yariv A., Xu Y., Lee R.K., Scherer A. // Opt. Lett. 1999. V. 24. N 11. P. 711.

[7] Gorodetsky M.L., Ilchenko V.S. // J. Opt. Soc. Am. B. 1999. V. 16. N 1. P. 147.

[8] Armani D.K., Kippenberg T.J., Spillane S.M., Vahala K.J. // Nature. 2003. V. 421. N 6926. P. 925.

[9] Moiseev E.S., Moiseev S.A. // Laser Phys. Lett. 2017. V. 14. N 1. P. 015202.

[10] Moiseev S.A., Gubaidullin F.F., Kirillov R.S., Latypov R.R., Perminov N.S., Petrovnin K.V., Sherstyukov O.N. // Phys. Rev. A. 2017. V. 95. N 1. P. 012338.

[11] Moiseev S.A., Gerasimov K.I., Latypov R.R., Perminov N.S., Petrovnin K.V., Sherstyukov O.N. // Sci. Rep. 2018. V. 8. N 1. P. 3982.

[12] Kurizki G., Bertet P., Kubo Y., Mфlmer K., Petrosyan D., Rabl P., Schmiedmayer J. // Proc. Nat. Acad. Sci. 2015. V. 112. P. 3866.

[13] Моисеев С.А., Андрианов С.Н. // Опт. и спектр. 2016. T. 121. № 6. С. 954; Моисеев С.А., Андрианов С.Н., Моисеев Е.С. // Опт. и спектр. 2013. Т. 115. № 3. С. 406.

[14] Perminov N.S., Tarankova D.Yu., Moiseev S.A. // arXiv preprint. 2017. arXiv:1711.07014; Perminov N.S., Moiseev S.A. // arXiv preprint. 2017. arXiv:1706.00592.

[15] Kockum A.F., Johansson G., Nori F. // Phys. Rev. Lett. 2018. V. 120. N 14. P. 140404.

[16] Moiseev S.A., Kröll S. // Phys. Rev. Lett. 2001. V. 87. N 17. P. 173601.

[17] Moiseev S.A. // J. Phys. B. 2007. V. 40. N 19. P. 3877.

[18] Tittel W., Afzelius M., Chaneliére T., Cone R., Kröll S., Moiseev S., Sellars M. // Las. Photonics Rev. 2009. V. 4. N 2. P. 244.

[19] De Riedmatten H., Afzelius M., Staudt M.U., Simon C., Gisin N. // Nature. 2008. V. 456. N 7223. P. 773.

[20] Moiseev S.A., Andrianov S.N., Gubaidullin F.F. // Phys. Rev. A. 2010. V. 82. N 2. P. 022311.

[21] Afzelius M., Simon C. // Phys. Rev. A. 2010. V. 82. N 2. P. 022310

[22] Walls D.F., Milburn G.J. Quantum Optics. Springer Science \& Business Media. 2007.

[23] Gorshkov A.V., André A., Lukin M.D., Sørensen A.S. // Phys. Rev. A. 2007. V. 76. N 3. P. 033804. 